\title{
Surgical resection for advanced thymic malignancy with pulmonary hilar invasion using hemi-clamshell approach
}

\author{
Ayako Fujiwara, Soichiro Funaki, Naoko Ose, Takashi Kanou, Ryu Kanzaki, Masato Minami, \\ Yasushi Shintani \\ Department of General Thoracic Surgery, Osaka University Graduate School of Medicine, Suita, Osaka, Japan \\ Contributions: (I) Conception and design: A Fujiwara, Y Shintani; (II) Administrative support: None; (III) Provision of study materials or patients: Y \\ Shintani; (IV) Collection and assembly of data: S Funaki, N Ose, T Kanou, R Kanzaki, M Minami; (V) Data analysis and interpretation: Y Shintani; (VI) \\ Manuscript writing: All authors; (VII) Final approval of manuscript: All authors. \\ Correspondence to: Yasushi Shintani, MD, PhD. Department of General Thoracic Surgery, Osaka University Graduate School of Medicine, 2-2-L5, \\ Yamadaoka, Suita, Osaka 565-0871, Japan. Email: yshintani@thoracic.med.osaka-u.ac.jp.
}

\begin{abstract}
Background: The hemi-clamshell (HCS) approach provides a wide anterior view of the mediastinum as well as outstanding exposure of the pulmonary hilum. Here, we evaluated the utility and outcomes of this approach in cases of advanced thymic malignancy with hilar invasion.

Methods: We performed a retrospective analysis of 14 patients with thymic epithelial malignancy surgically resected with an HCS approach. All required lung resection because of suspected pulmonary hilar vessel invasion.

Results: Histological findings showed that 8 patients had a thymoma and 6 a thymic carcinoma. Thirteen patients underwent lung resection, a lobectomy or bilobectomy in 8 and wedge resection in 5, while 1 had an exploratory thoracotomy. Seven patients with a thymoma underwent resection of disseminated lesions and 8 of 10 who underwent phrenic nerve resection received diaphragmatic plication through an HCS procedure. There were no postoperative mortalities. Macroscopic complete surgical resection was achieved in 13 cases.

Conclusions: An HCS approach was helpful for lung resection performed for advanced thymic malignancy with hilar invasion by providing multiple access paths to the tumor and hilum, allowing for a sufficient surgical margin. Furthermore, it was useful for resection of disseminated lesions and diaphragmatic plication.
\end{abstract}

Keywords: Thymic malignancy; hemi-clamshell approach (HCS approach); pulmonary resection

Submitted Sep 19, 2018. Accepted for publication Nov 15, 2018.

doi: $10.21037 /$ jtd.2018.11.73

View this article at: http://dx.doi.org/10.21037/jtd.2018.11.73

\section{Introduction}

Epithelial tumors of the thymus, including thymoma, thymic carcinoma, and thymic carcinoid, are the most commonly encountered type of mediastinal tumors $(1,2)$. They occasionally invade or disseminate to surrounding tissues, such as the aorta, superior vena cava (SVC), pericardium, or diaphragm, which makes complete resection difficult, though rarely metastasize to distant extra-thoracic sites (3). When invasion is located near the center of the body, resection can be performed via a median approach. However, when a lobectomy is needed because of invasion to the hilar, such an approach is not adequate, because the tumor itself blocks the surgical view and complicates manipulation.

The hemi-clamshell (HCS) approach is a surgical method that includes a partial median sternotomy and anterior thoracotomy, and may also include cervical extension (4), which provides outstanding exposure to the apex of the chest. Thus, large and apical intrathoracic tumors, cervicothoracic masses including superior sulcus tumors, and trauma to the subclavian or innominate vessels are the most common indications for HCS (5). Furthermore, 

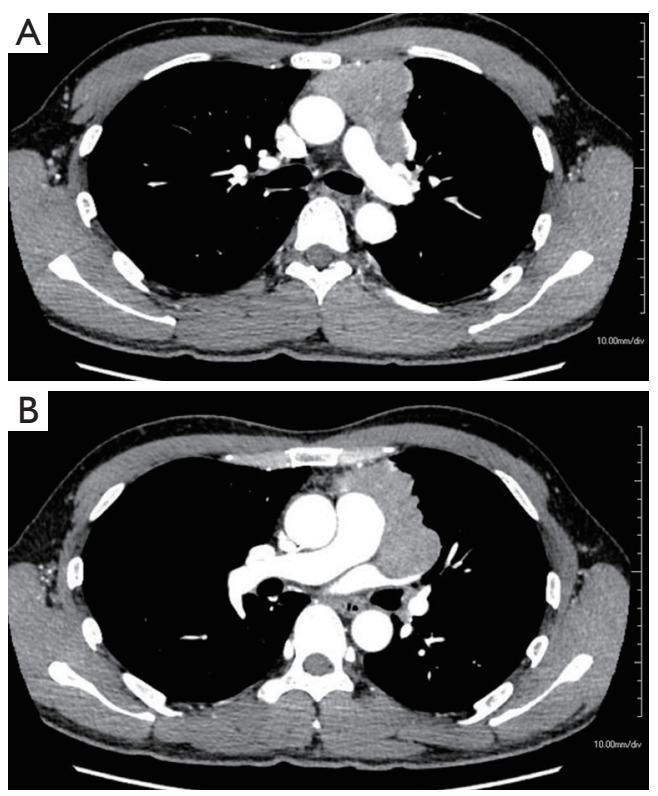

Figure 1 CT scan images from a representative case. A mediastinal tumor suspected of invading the pulmonary trunk (A) and left upper pulmonary vein (B), together with the pericardium or ascending aorta.

this approach also provides a wide anterior view of the mediastinum, making it useful for mediastinal surgery, especially in cases of advanced malignancy. Unfortunately, there are few case reports showing adaptation and advantages of this approach for thymic malignancy. We have used an HCS approach for complete resection of advanced thymic malignancy when preoperative imaging suggests tumor invasion of the unilateral pulmonary hilum.

For this study, we retrospectively investigated 14 patients who underwent an operation with an HCS approach for advanced thymoma or thymic cancer with lung invasion in order to evaluate its advantages.

\section{Methods}

\section{Patients}

This was a retrospective analysis of 14 patients with thymic epithelial malignancy who underwent surgical resection between 2010 and 2016 at Osaka University Hospital. Approval for this study was provided by our Institutional Review Board (\#10026-3), and written informed consent for surgery and this analysis was obtained from each patient.

\section{Treatment strategies}

All patients with a thymic tumor underwent chest computed tomography (CT), while some also received chest magnetic resonance imaging (MRI) to determine the degree of invasion to surrounding organs. When a tumor showed only contact with or slight invasion of a large vessel, surgical resection was immediately planned. If complete resection was not expected because of severe tumor invasion to surrounding organs, a tumor biopsy using CT-guided or video-assisted thoracic surgery (VATS) was performed, then induction therapy was scheduled according to the diagnosis. For the thymoma patients, chemotherapy, which usually included cisplatin and doxorubicin, was given, with steroid pulse therapy also utilized in some. For the thymic carcinoma patients, radiotherapy was generally performed concurrently with chemotherapy. Most of the chemotherapy regimens included cisplatin and docetaxel, though carboplatin, paclitaxel, and etoposide were given instead to some patients. Following induction therapy, resectability was judged again using chest CT findings, then surgical resection was planned within 4 to 8 weeks after completion of therapy. Thymoma patients with disseminated lesions were considered to be indicated for surgery, while disseminated lesions in thymic carcinoma patients were thought to be a contraindication for surgery and chemoradiotherapy was continued in those cases. Most patients with a thymic tumor underwent surgical resection via a median approach, while an HCS approach was selected for those who required a lobectomy, because of suspected invasion of the lung parenchyma or hilar vessels.

\section{Representative case}

Chest CT findings of a representative case are shown in Figure 1. The patient was a 41-year-old male with myasthenia gravis. A mediastinal tumor $7 \mathrm{~cm}$ in diameter was found on the left side, and invasion to the pulmonary trunk and left upper pulmonary vein together with the pericardium or ascending aorta was suspected. We performed an extended thymectomy via an HCS approach with excision of the tumor, left upper lobe, disseminated lesions, pericardium, and left phrenic nerve. Although tumors with invasion to large vessels of the mediastinum can be resected via a median approach, HCS was needed in this case to resect the mediastinal tumor showing hilar invasion. 


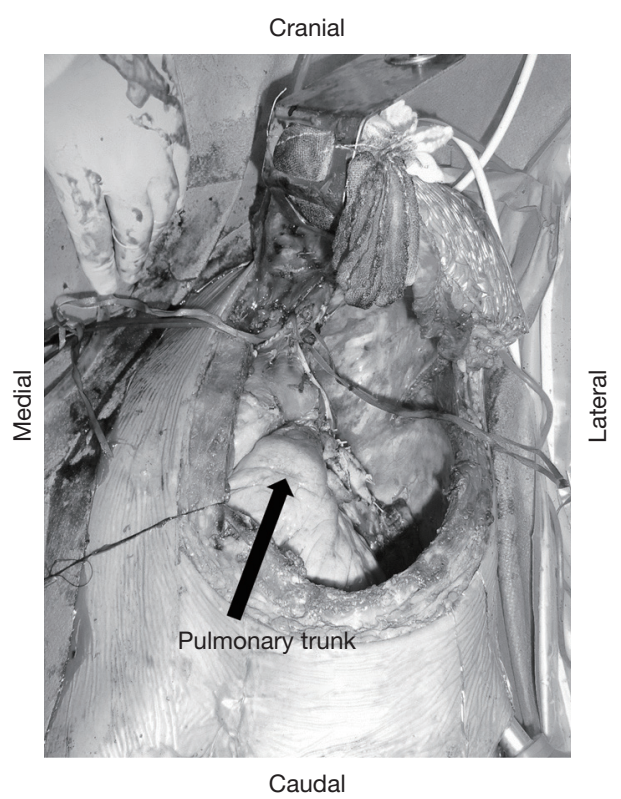

Figure 2 Representative surgical view offered by the HCS approach, by which a wide antero-lateral view of the mediastinal vascular complex can be obtained. HCS, hemi-clamshell.

\section{Operative procedure}

In general, the operative procedure used in the present cases was based on our previous report regarding an HCS approach for advanced primary lung cancer $(6,7)$. The patient is placed in a semi-lateral decubitus position and secured firmly to the surgical bed to allow for rotation. Prior to beginning this approach, we often use thoracoscopic inspection for confirmation of tumor invasion extending to the hilum as well as to rule out dissemination in patients with thymic cancer. A cutaneous incision with an $\mathrm{L}$ or inverted $\mathrm{L}$ shape is used so as to avoid a T-shape. Thoracic access is then obtained with an upper median sternotomy, and a fourth or fifth intercostal thoracotomy, followed by retraction of the flap of the anterior chest wall in a vertical direction (Figure S1). When tumor involvement includes the apex of the chest such as the subclavian vessels, we perform a pulmonary resection via an HCS approach after dividing the first costal cartilage to provide greater lift of the flap of the chest wall, which allows lateral access to the subclavian vessels (7). For cases with deep pleural dissemination, a fifth intercostal thoracotomy is selected. In most of the present cases, an ipsilateral collar incision is added to obtain a wider view and prevent skin tightening. A representative surgical field obtained with our HCS method is shown in Figures 2 and $S 2$.

\section{Results}

At Osaka University Hospital, 144 patients underwent surgical resection for thymic malignancies between 2010 and 2016. Of those, 14 (9.7\%), including 5 males and 9 females, underwent a radical resection via an HCS approach. Clinical information for each patient is summarized in Table 1. Median age at the time of surgery was 52.9 years (range, 30-77 years). Histological findings showed that 8 patients had a thymoma and 6 a thymic carcinoma. Of the thymoma patients, 2 were classified as World Health Organization (WHO) type B1, 2 were B2, 2 were B3, and 2 were combined B2 and B3. The thymic carcinoma group consisted of 3 with a squamous cell carcinoma, 1 with a large cell neuroendocrine carcinoma, 1 with a poorly differentiated carcinoma, and 1 with a mucoepidermoid carcinoma. The median size of all tumors was $81.5 \mathrm{~mm}$ (41$124 \mathrm{~mm}$ ). At the time of initial staging, thymic carcinoma patients were given a diagnosis of Masaoka-stage III and thymoma patients were IVa. A list of organs with clinically suspected infiltration in the patients is shown in Table 2.

All patients underwent tumor resection with a thymectomy, with an extended thymectomy performed for 2 with myasthenia gravis. In 4 cases, we initially intended to perform a median full sternotomy and then later converted to an HCS approach, resulting in a T-shape skin incision (Table 2). A fourth intercostal thoracotomy was performed in 9 and a fifth in 5 for the HCS approach. As shown in Table 3, 13 of the 14 patients underwent lung resection, with a lobectomy performed in 7 , a bilobectomy in 1 , and a wedge resection in 5 , while the remaining patient underwent an exploratory thoracotomy. Organs resected included the phrenic nerve in 10 , pericardia in 9, left innominate vein in 6, vagal nerve in 2, aortic arch in 1, and SVC in 1, with overlap in some cases. Eight of 10 patients with phrenic nerve resection underwent diaphragmatic plication through an HCS approach to prevent paradoxical diaphragmatic movement. All thymoma patients, except the 1 who underwent an exploratory thoracotomy, had disseminated lesions resected via an HCS approach incision. The median operative time was 417 minutes (203-678 minutes) and blood loss was $1,440 \mathrm{~mL}(330-5,500 \mathrm{~mL})$. Four patients had cardiovascular complications that did not require treatment, including 2 with tachycardia, 1 with atrial fibrillation, and 1 with pericardial effusion. One patient had intrapleural 
Table 1 Patient characteristics

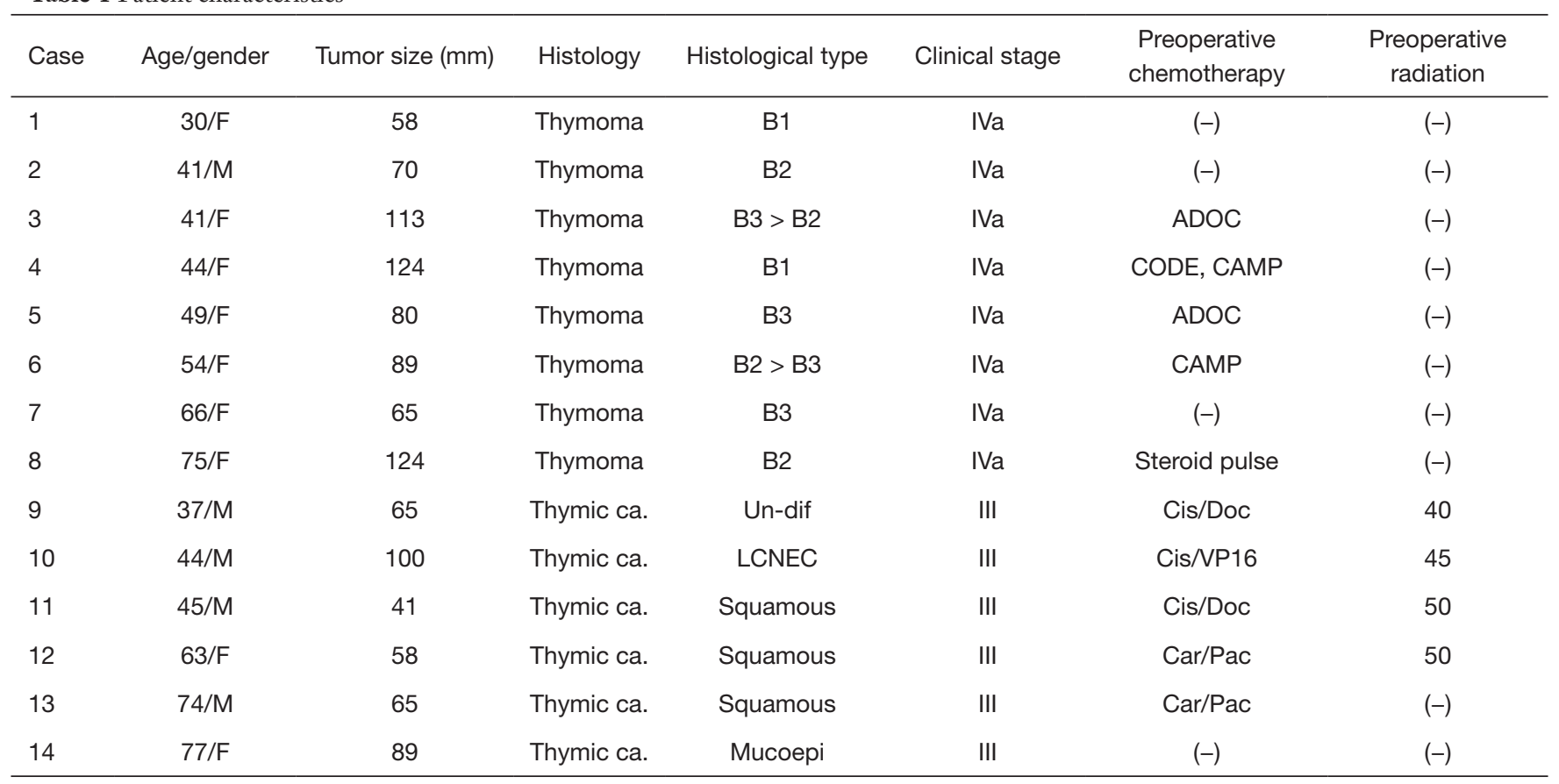

Thymic ca., thymic carcinoma; un-dif, undifferentiated carcinoma; LCNEC, large cell neuroendocrine carcinoma; squamous, squamous cell carcinoma; Mucoepi, mucoepidermoid carcinoma; ADOC, Adriamycin, cisplatin (CDDP), vincristine (VCR) and cyclophosphamide; CODE, CDDP, VCR, doxorubicin (DXR) and etoposide; CAMP, CDDP, DXR, methylprednisolone; Cis, CDDP; Doc, docetaxel; VP16, etoposide; Car, carboplatin; Pac, paclitaxel; PR, partial response; SD, stable disease.

Table 2 Clinically suspected infiltrated organs

\begin{tabular}{|c|c|c|c|c|c|c|c|}
\hline Case & Histology & \multicolumn{5}{|c|}{ Clinically infiltrated organ } & $\begin{array}{l}\text { Converted to HCS-A } \\
\text { from sternotomy }\end{array}$ \\
\hline 1 & Thymoma & $\bullet$ & $\bullet$ & $\bullet$ & $\bullet$ & & \\
\hline 2 & Thymoma & $\bullet$ & $\bullet$ & $\bullet$ & & & \\
\hline 3 & Thymoma & $\bullet$ & & $\bullet$ & & Right atrium, SVC & $\bullet$ \\
\hline 5 & Thymoma & $\bullet$ & $\bullet$ & - & $\bullet$ & Left BCV & \\
\hline 6 & Thymoma & $\bullet$ & & $\bullet$ & $\bullet$ & Left BCV & $\bullet$ \\
\hline 7 & Thymoma & $\bullet$ & $\bullet$ & $\bullet$ & & Left BCV & \\
\hline 8 & Thymoma & $\bullet$ & $\bullet$ & $\bullet$ & $\bullet$ & Left BCV & \\
\hline 12 & Thymic ca. & $\bullet$ & $\bullet$ & & $\bullet$ & Left BCV, chest wall & \\
\hline 13 & Thymic ca. & $\bullet$ & $\bullet$ & & $\bullet$ & & \\
\hline 14 & Thymic ca. & $\bullet$ & $\bullet$ & & & & \\
\hline
\end{tabular}

The black dot indicates the suspicion of inflation to each organ. Thymic ca., thymic carcinoma; hilar, hilar vessels; pleural, pleural dissemination; SVC, superior vena cava; BCV, brachiocephalic vein; HCS-A, hemi-clamshell approach. 
Table 3 Operative procedure of the lung and surgical results

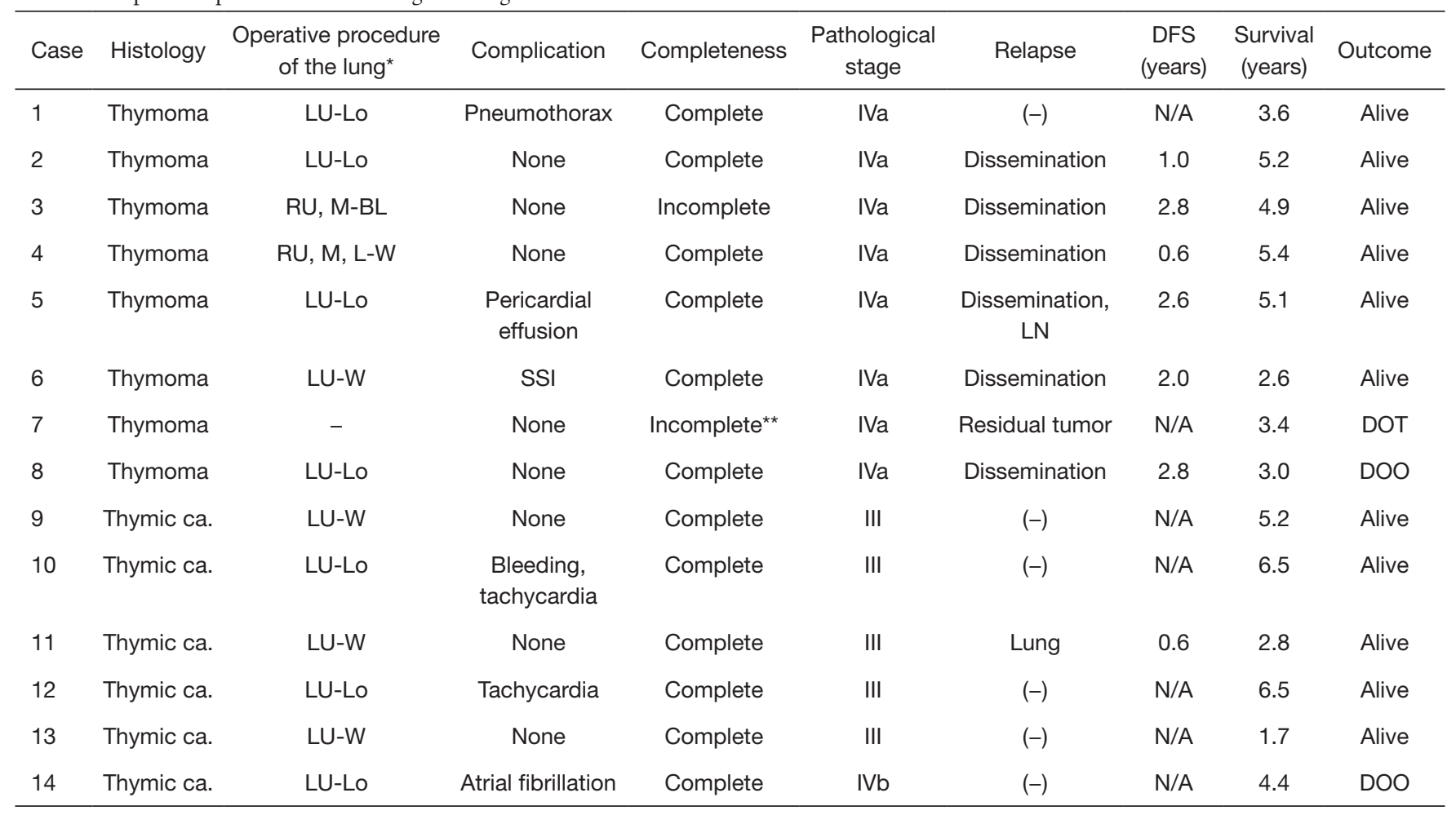

*, L, left; R, right; U, upper; M, middle; L, lower; Lo, lobectomy; W, wedge resection; BL, bi-lobectomy. ${ }^{* *}$, Case 7 was performed exploratory thoracotomy only. LN, lymph node; DFS, disease free survival; N/A, not applicable; SSI, surgical site infection; DOT, dead of tumor; DOO, dead of other causes; thymic ca., thymic carcinoma.

bleeding after the operation for which surgical intervention was performed. The median postoperative hospital stay was 21.0 days (10-30 days). There were no postoperative mortalities.

Macroscopic complete surgical resection was achieved in 13 patients. Complete resection could not be achieved in Patient 7, who underwent an exploratory thoracotomy because of tumor infiltration into the pulmonary trunk with pericardial dissemination. Pathologically, 5 patients were given a diagnosis of Masaoka-stage III, 8 stage IVa, and 1 stage $\mathrm{IVb}$ because of metastasis to the anterior mediastinal lymph nodes. All patients with thymic cancer underwent microscopic complete resection to the resection margin, while 7 with a thymoma underwent macroscopic complete resection of disseminated lesions.

The median follow-up period was 5.4 years. At the time of analysis, 3 patients had died, 1 from thymoma recurrence and 2 from other diseases (Table 3). Of the 11 living patients, 6 thymoma and 1 thymic carcinoma patients were alive with relapse.

\section{Discussion}

To perform a secure surgical procedure in cases of advanced malignancy, wide exposure is mandatory and of primary importance. Although a median sternotomy is the gold standard for access to the mediastinum, that approach does not provide an adequate view for accessing the upper and lower chest cavity or deeper inside, and the optimal surgical approach for advanced thymic malignancy cases remains a point of discussion (8). The HCS approach has been shown to provide excellent exposure of tumor-infiltrated organs $(9,10)$. At our institution, we use such a technique for patients with thymic malignancy who require a lobectomy because of suspected hilar vessel invasion. For successful lung resection, the HCS approach is helpful by providing multiple points of access to the tumor and hilum, as well as the interlobar surface.

Among the present cohort, a lobectomy was avoided in 5 patients who underwent a wedge resection, because intraoperative findings showed that the tumor had invaded the lung parenchyma but not hilar vessels. We have found 
that even in patients who undergo a wedge resection of the lung, the wide view provided by this approach is helpful for a safe lung resection procedure with a sufficient surgical margin, as well as providing multiple access options to the tumor and hilum. We have also found that an HCS approach is useful for resection of deep pleural disseminated lesions and also suitable for diaphragmatic plication in cases with phrenic nerve resection, which are advantages frequently needed for successful resection of advanced thymic malignancy.

Some have reported that surgical debulking is an acceptable procedure for an invasive thymoma, because of the potential for favorable outcome (11). We agree with that concept and prefer conservative treatment with resection of visible disseminated nodules by use of a partial pleurectomy in patients classified as Masaoka stage IVa. While macroscopic total resection appears to be a promising prognostic factor for stage IVa, a pleuropneumonectomy also seems to be warranted for a small number of highly selected patients with an advanced or recurrent thymoma extensively involving the pleural space (12). Thus, we often perform a partial or subtotal pleurectomy, and remove as much of the disseminated tumor as possible in patients with a stage IVa thymoma using HCS.

A previous study reported that due to the anatomical course of the phrenic nerve along the mediastinal pleura adjacent to the thymus at the level of the upper mediastinum, it is involved in up to $33 \%$ of advanced stage thymoma cases (13). When the involvement is unilateral, phrenic nerve resection is possible and may be necessary, because it is associated with a loss of vital capacity of up to one third (14). When the phrenic nerve is not detected in the mediastinal pleura due to tumor invasion, we basically resect that with the tumor and then perform diaphragmatic plication. When thymic cancer has invaded the phrenic nerve, we routinely resect it along with the tumor.

There are some disadvantages associated with the HCS approach, such as need for an extended incision, which may lead to a flailed chest, rib fracture, or neuralgia (4). However, we have never encountered postoperative paradoxical chest wall motion or rib fracture in such patients treated at our institution. Additionally, this approach usually includes an L-shaped sternum disconnection technique in contrast to a T-shape, which may be an advantage to maintain the rigidity of the chest wall. As for potential rib fracture, the wide operative field can prevent excess pressure on bones, causing breakage. As for neuralgia and postoperative wound pain, we usually administer a non-steroid anti-inflammatory drug (NSAID) after the epidural anesthesia administration has finished. All in the present cohort stopped medication for postoperative wound pain at an average of 1.5 months after the operation, with the exception of 1 patient who continued taking pregabalin for up to 3 months. Thus, pain was reduced despite the length of the wound. A potential limitation of this approach is that it does not allow full access to the posterior thoracic cavity. We previously reported a patient with a huge thymoma with multiple pleural dissemination, for whom we performed a pleuropneumonectomy (12). For such cases, a one-step surgical procedure using a median sternotomy in combination with a posterolateral thoracotomy may be an alternative. When the thymoma has invaded hilar vessels with dissemination in the posterior thoracic cavity, we often add support with video thoracic surgery to the HCS approach for a pleurectomy.

Complete surgical resection and Masaoka stage are important prognostic factors for thymic malignancy $(15,16)$. In our study, complete resection was achieved in 13 of 14 patients despite the fact that all were in an advanced stage. After a median follow-up period of 5.4 years, 11 were alive, including 5 of 6 patients treated for thymic cancer and 1 of 8 for a thymoma without recurrence. Six patients with a thymoma had recurrence in the pleural cavity, and underwent a repeated resection or radiotherapy for pleural dissemination, thus the disease is considered to be well controlled. Although our study population was small and the observation period not long, our results of surgical intervention with curative intent for patients with advanced thymic malignancy are encouraging.

In conclusion, we found that the HCS approach provides a wide surgical view, making it easier to access tumor infiltrated organs. Furthermore, it was especially helpful for situations requiring lung hilum manipulation, resection of disseminated lesions, or diaphragmatic plication. We consider this approach to be an attractive option for managing advanced thymic malignancy.

\section{Acknowledgements}

None.

\section{Footnote}

Conflicts of Interest: The authors have no conflicts of interest to declare.

Ethical Statement: Approval for this study was provided by 
our Institutional Review Board (\#10026-3), and written informed consent was obtained from the patient for publication of this manuscript and any accompanying images.

\section{References}

1. Detterbeck FC, Parsons AM. Thymic tumors. Ann Thorac Surg 2004;77:1860-9.

2. Okumura M, Shiono H, Minami M, et al. Clinical and pathological aspects of thymic epithelial tumors. Gen Thorac Cardiovasc Surg 2008;56:10-6.

3. Park BJ, Bacchetta M, Bains MS, et al. Surgical management of thoracic malignancies invading the heart or great vessels. Ann Thorac Surg 2004;78:1024-30.

4. Masaoka A, Ito Y, Yasumitsu T. Anterior approach for tumor of the superior sulcus. J Thorac Cardiovasc Surg 1979;78:413-5.

5. Korst RJ, Burt ME. Cervicothoracic tumors: results of resection by the "hemi-clamshell" approach. J Thorac Cardiovasc Surg 1998;115:286-94; discussion 294-5.

6. Ohta M, Hirabayasi H, Shiono H, et al. Hemi-clamshell approach for advanced primary lung cancer. Thorac Cardiovasc Surg 2004;52:200-5.

7. Shintani Y, Kanzaki R, Kawamura T, et al. Surgical resection for advanced lung cancer using the hemiclamshell approach. Interact Cardiovasc Thorac Surg 2017;25:462-8.

8. Limmer S, Merz H, Kujath P. Giant thymoma in the anterior-inferior mediastinum. Interact Cardiovasc Thorac

Cite this article as: Fujiwara A, Funaki S, Ose N, Kanou T, Kanzaki R, Minami M, Shintani Y. Surgical resection for advanced thymic malignancy with pulmonary hilar invasion using hemi-clamshell approach. J Thorac Dis 2018;10(12):64756481. doi: $10.21037 /$ jtd.2018.11.73
Surg 2010;10:451-3.

9. Takenaka T, Ishida T, Handa Y, et al. Ectopic thymoma presenting as a giant intrathoracic mass: a case report. J Cardiothorac Surg 2012;7:68.

10. Saito T, Makino T, Hata Y, et al. Giant thymoma successfully resected via anterolateral thoracotomy: a case report. J Cardiothorac Surg 2015;10:110.

11. Attaran S, Acharya M, Anderson JR, et al. Does surgical debulking for advanced stages of thymoma improve survival? Interact Cardiovasc Thorac Surg 2012;15:494-7.

12. Shintani Y, Kanzaki R, Kusumoto H, et al. Pleuropneumonectomy for a large thymoma with multiple pleural dissemination using median sternotomy followed by posterolateral thoracotomy. Surg Case Rep 2015;1:75.

13. Venuta F, Rendina EA, Longo F, et al. Long-term outcome after multimodality treatment for stage III thymic tumors. Ann Thorac Surg 2003;76:1866-72.

14. Yano M, Sasaki H, Moriyama S, et al. Preservation of Phrenic Nerve Involved by Stage III Thymoma. Ann Thorac Surg 2010;89:1612-9.

15. Lee CY, Bae MK, Park IK, et al. Early Masaoka stage and complete resection is important for prognosis of thymic carcinoma: a 20-year experience at a single institution. Eur J Cardiothorac Surg 2009;36:159-62; discussion 163.

16. Kondo K, Van Schil P, Detterbeck FC, et al. The IASLC/ITMIG Thymic Epithelial Tumors Staging Project: proposals for the $\mathrm{N}$ and $\mathrm{M}$ components for the forthcoming (8th) edition of the TNM classification of malignant tumors. J Thorac Oncol 2014;9:S81-7. 


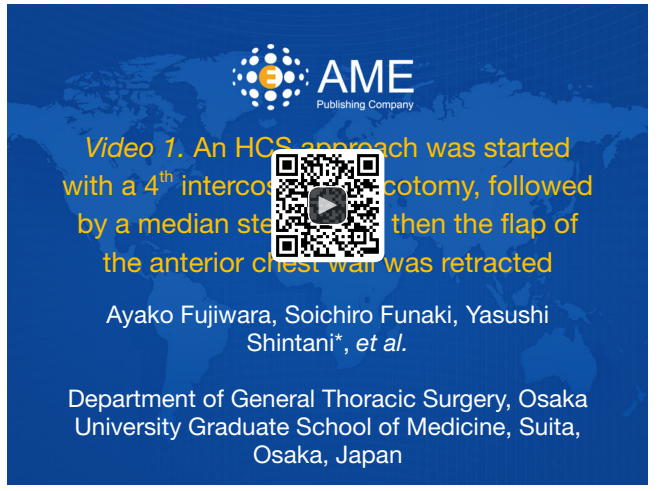

Figure S1 An HCS approach was started with a $4^{\text {th }}$ intercostal thoracotomy, followed by a median sternotomy, then the flap of the anterior chest wall was retracted (17). HCS, hemi-clamshell. Available online: http://www.asvide.com/article/view/29195

\section{References}

17. Fujiwara A, Funaki S, Ose N, et al. An HCS approach was started with a 4th intercostal thoracotomy, followed by a median sternotomy, then the flap of the anterior chest wall was retracted. Asvide 2018;5:933. Available online: http://

\section{A?: AME}

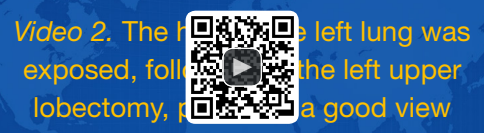

Ayako Fujiwara, Soichiro Funaki, Yasushi

Shintani*, et al.

Department of General Thoracic Surgery, Osaka

University Graduate School of Medicine, Suita,

Osaka, Japan

Figure S2 The hilar of the left lung was exposed, followed by the left upper lobectomy, providing a good view (18).

Available online: http://www.asvide.com/article/view/29196

www.asvide.com/article/view/29195

18. Fujiwara A, Funaki S, Ose N, et al. The hilar of the left lung was exposed, followed by the left upper lobectomy, providing a good view. Asvide 2018;5:934. Available online: http://www.asvide.com/article/view/29196 